PROCEEDINGS OF THE

AMERICAN MATHEMATICAL SOCIETY

Volume 137, Number 4, April 2009, Pages 1215-1222

S 0002-9939(08)09674-3

Article electronically published on October 2, 2008

\title{
PFISTER'S THEOREM FOR ORTHOGONAL INVOLUTIONS OF DEGREE 12
}

\author{
SKIP GARIBALDI AND ANNE QUÉGUINER-MATHIEU
}

(Communicated by Martin Lorenz)

\begin{abstract}
We use the fact that a projective half-spin representation of $\operatorname{Spin}_{12}$ has an open orbit to generalize Pfister's result on quadratic forms of dimension 12 in $I^{3}$ to orthogonal involutions.
\end{abstract}

In his seminal paper $[\mathrm{Pf}]$, Pfister proved strong theorems describing quadratic forms of even dimension $\leq 12$ that have trivial discriminant and Clifford invariant, i.e., that are in $I^{3}$. His results have been extended to quadratic forms of dimension 14 in $I^{3}$ by Rost; see $[\mathrm{R}$ or $\mathrm{GaC}$. One knows also extensions of these theorems where quadratic forms are replaced by central simple algebras with orthogonal involution, except in degree 12. The purpose of this paper is to complete this picture by giving the extension in the degree 12 case. The principle underlying the quadratic forms results and our extension is that a projective half-spin representation of $\mathrm{Spin}_{n}$ for even $n$ has an open orbit precisely for $n \leq 14$; cf. [R], [ $]$, and [SK]. However, we make use of this principle in a new way; Rost's original method was to use it to directly provide a surjection in Galois cohomology.

Let us first recall what is already known. We consider quadratic forms $q$ of even dimension (resp. central simple algebras with orthogonal involution $(A, \sigma)$ of even degree) with trivial discriminant and Clifford invariant. If $q$ has dimension $<8$, then $q$ is hyperbolic by the Arason-Pfister Hauptsatz [L, X.5.1]. This also holds in the nonsplit case: if $A$ has degree $<8$, then $\sigma$ is hyperbolic; see e.g. $\mathrm{GaO}$, 1.4] or [Q, 4.4]. If $q$ has dimension 8 , then $q$ is similar to a 3-Pfister form [L, X.5.6]; if $A$ has degree 8 , then $(A, \sigma)$ is isomorphic to a tensor product $\bigotimes_{i=1}^{3}\left(Q_{i}, \sigma_{i}\right)$ of quaternion algebras with orthogonal involution [KMRT, 42.11]. If $A$ has degree 10 or 14 , then $A$ is necessarily split [Ga, 1.5$]$, so there is no interesting generalization of the theorem on quadratic forms. The remaining case is where $q$ has dimension 12 , where Pfister proved that $q$ is isomorphic to $\phi \otimes \psi$ for some 1-Pfister $\phi$ and 6dimensional form $\psi$ with trivial discriminant; see [Pf] pp. 123, 124] or [Ga C, 17.13]. In Theorem 3.1 below, we prove an analogous statement for $(A, \sigma)$ in case $A$ has degree 12. We do not use Pfister's theorem on 12-dimensional quadratic forms in our proof, so we obtain his result as a corollary (Corollary 3.2).

This paper has three sections. First, we study quadratic extensions of algebras with involution, and in particular orthogonal extensions of unitary involutions, as considered in [BP, App. 2], ET, §3], and [QT, 2.14]. Second, we show how to

Received by the editors April 10, 2008.

2000 Mathematics Subject Classification. Primary 20G15; Secondary 16W10, 11 E04.

(C)2008 American Mathematical Society

Reverts to public domain 28 years from publication 
construct an algebra with orthogonal involution that has trivial discriminant and Clifford invariant from any exponent 2 algebra with unitary involution. Third, we prove that in degree 12 , this construction produces every central simple algebra with orthogonal involution that has trivial discriminant and Clifford invariant.

In the language of linear algebraic groups, our results are as follows. Our construction takes a group of type ${ }^{2} A_{n-1}$ (e.g., $\mathrm{SU}_{n}$ ) and produces a group of type ${ }^{1} D_{n}$ (e.g., $\operatorname{Spin}_{2 n}$ ). Our Theorem 3.1 shows that, over a field $F$, every algebraic group of type $D_{6}$ with a half-spin representation defined over $F$ is obtained by our construction.

Global conventions. We work over a base field $F$ of characteristic $\neq 2$. We use the notation and basic language of [KMRT]. A few main points are: We extend the notions of central simple algebra and Brauer-equivalence over a quadratic field extension $K / F$ in an obvious way to include also the case where $K$ is the split étale algebra $F \times F$. For $a \in A^{\times}$, we write $\operatorname{Int}(a)$ for the map $x \mapsto a x a^{-1}$ on $A$.

Let $(A, \sigma)$ be a central simple algebra of even degree with orthogonal involution. The (signed) discriminant of $\sigma$ is an element of $F^{\times} / F^{\times 2}=H^{1}\left(F, \boldsymbol{\mu}_{2}\right)$. If the discriminant of $\sigma$ is trivial, then the even Clifford algebra of $(A, \sigma)$ is a product $C_{+} \times C_{-}$of central simple algebras such that $\left[C_{+}\right]-\left[C_{-}\right]$equals $[A]$ in the Brauer group of $F$ (see for instance [KMRT, Th. 9.12]). The Clifford invariant is the class of $\left[C_{+}\right]$or $\left[C_{-}\right]$in the quotient $\operatorname{Br}(F) /[A]$.

For an $n$-dimensional quadratic form $q$ over $F$, we write $\mathrm{Ad}_{q}$ for the split algebra with adjoint involution $\left(M_{n}(F), \operatorname{ad}_{q}\right)$.

\section{QuAdRATIC EXTENSIONS OF ALGEBRAS With INVOLUTION}

1.1. Let $(A, \sigma)$ be a central simple $F$-algebra with involution, and suppose that $A$ contains a $\sigma$-stable quadratic étale $F$-algebra $K$. One can write $K$ as $F[\delta] /\left(\delta^{2}-d\right)$ for some $d \in F^{\times}$, so this hypothesis is equivalent to saying: there is a noncentral $\delta \in A$ such that $\sigma(\delta)= \pm \delta$ and $\delta^{2} \in F^{\times}$. The centralizer $B$ of $K$ in $A$ is a central simple $K$-algebra Brauer-equivalent to $A \otimes K$, and of degree $\frac{1}{2} \operatorname{deg}(A)$. We write $\tau$ for the involution on $B$ induced by $\sigma$, and we say that $(A, \sigma)$ is a quadratic extension of $(B, \tau)$. Note in particular that if $A$ and $B$ are division algebras, then $A$ is a quadratic internal extension of $B$ as in Dieudonné $[D$.

Example 1.2. Let $(Q, \gamma)$ be a quaternion $F$-algebra, with involution of the first kind. For any $\gamma$ symmetric or skew-symmetric pure quaternion $i,(Q, \gamma)$ is a quadratic extension of $\left(F(i), \gamma_{\mid F(i)}\right)$.

Example 1.3. Consider now another algebra with involution of the first kind $\left(B_{0}, \tau_{0}\right)$ over $F$; the tensor product $(A, \sigma)=\left(B_{0}, \tau_{0}\right) \otimes(Q, \gamma)$ is a quadratic extension of $(B, \tau)=\left(B_{0}, \tau_{0}\right) \otimes\left(F(i), \gamma_{\mid F(i)}\right)$.

Example 1.4. In particular, if a quadratic form $q$ decomposes as $\psi \otimes\langle 1,-d\rangle$, the algebra with involution $\operatorname{Ad}_{q}$ is a quadratic extension of $\operatorname{Ad}_{\psi} \otimes_{F}\left(F(\sqrt{d}),{ }^{-}\right)$.

1.5. We will now restrict our attention to extensions of $K / F$-unitary involutions; that is, we assume $K=F[\delta]$ with $\delta^{2}=d$ and $\sigma(\delta)=-\delta$. If $A$ is not split, $\sigma$ is not orthogonal, or $\sigma$ has even Witt index (e.g., if $\sigma$ is anisotropic), then by [BST, 3.3], the existence of such a $\delta$ is equivalent to the statement $\sigma$ is hyperbolic over $F$ or a quadratic extension of $F$. 
One remarkable fact about the situation in 1.1 is that the involution $\sigma$ is uniquely determined by its restriction $\tau$ :

Lemma 1.6. Let $(A, \sigma)$ be a quadratic extension of $(B, \tau)$, and assume that $\tau$ is $K / F$ unitary. The only involutions on $A$ that agree with $\tau$ on $B$ are $\sigma$ and $\operatorname{Int}(\delta) \circ \sigma$. In particular, there is a unique orthogonal (resp. symplectic) involution on $A$ that is $\tau$ on $B$.

Proof. If $\tau$ is $K / F$ unitary, then in particular it acts on $F$ as the identity, and every involution on $A$ that agrees with $\tau$ on $B$ is of the first kind. Let $\sigma^{\prime}$ be such an involution, and pick $a \in A^{\times}$such that $\sigma(a)= \pm a$ and $\sigma^{\prime}=\operatorname{Int}(a) \circ \sigma$. Since $\sigma^{\prime}$ and $\sigma$ agree on $B$, the element $a$ centralizes $B$ and by the Double Centralizer Theorem belongs to $K$. If $\sigma(a)=a$, this means $a$ is in $F$ and $\sigma^{\prime}=\sigma$. Otherwise, $a$ is in $\delta F$ and $\sigma^{\prime}=\operatorname{Int}(\delta) \circ \sigma$ and the type of $\sigma^{\prime}$ is opposite that of $\sigma$.

Remark 1.7. It is obvious - both from the proof above and from Example 1.2 that the conclusion of Lemma 1.6 is no longer true if $\tau$ is of the first kind.

1.8. Lemma 1.6 shows that $\sigma$ is completely determined by its restriction to $B$. We now describe an explicit procedure to recover $(A, \sigma)$ from $(B, \tau)$ if we know the Brauer class of $A$ and the type of $\sigma$, thus proving:

Proposition 1.9. Let $K$ be a quadratic étale $F$-algebra and $(B, \tau)$ a central simple $K$-algebra with unitary $K / F$-involution. We assume $B$ has exponent at most 2 , and we fix a Brauer class $\beta \in \mathrm{Br}_{2}(F)$ whose restriction to $K$ is the class of $B$. There exists a unique orthogonal (resp. symplectic) quadratic extension $(A, \sigma)$ of $(B, \tau)$ with Brauer class $\beta$.

Note that, since $B$ has trivial corestriction, such a class $\beta$ exists, as we show in our proof.

Proof. We prove existence, beginning as in ET, pp. 380, 381]: Since $B$ has exponent 2 , it has an involution $\nu$ of the first kind of the desired type (orthogonal or symplectic). One can find an element $u \in B^{\times}$such that

$$
\nu(u)=\tau(u)=u \quad \text { and } \quad(\nu \tau)^{2}(x)=u x u^{-1} \quad(x \in B) .
$$

We define an $F$-algebra $A_{1}$ to be the vector space $B \oplus B z_{1}$ with multiplication rules

$$
z_{1}^{2}=u \quad \text { and } \quad z_{1} b=(\nu \tau)(b) z_{1} .
$$

It is a central simple $F$-algebra $[\mathrm{A}$, Chap. 11, Th. 10]. Moreover, the centralizer of $K$ in $A_{1}$ is $B$, so the class of $A_{1}$ is a Brauer class over $F$ whose restriction to $K$ is the class of $B$. Hence, it coincides with $\beta$ after scalar extension to $K$, and there is some choice of $\lambda \in F^{\times}$such that $A_{1} \otimes(K, \lambda)$ has Brauer class $\beta$.

Equations (1.10) only determine $u$ up to a central factor. By [KMRT, 13.41], replacing $u$ by $\lambda u$ in the construction above produces an algebra $A=B \oplus B z$ with Brauer class $\beta$.

This algebra $A$ is endowed with an involution $\sigma$ defined by

$$
\sigma\left(b_{1}+b_{2} z\right):=\tau\left(b_{1}\right)+z \tau\left(b_{2}\right) \quad\left(b_{1}, b_{2} \in B\right) .
$$

We show that this involution has the same type as $\nu$. Write $\operatorname{Sym}(A, \sigma)$ and $\operatorname{Sym}(B, \tau)$ for the subspaces of symmetric elements. Clearly,

$$
\operatorname{Sym}(A, \sigma)=\operatorname{Sym}(B, \tau) \oplus z \operatorname{Sym}(B, \nu) \text {. }
$$


So the dimension over $F$ of $\operatorname{Sym}(A, \sigma)$ is

$$
\operatorname{dim}_{F} \operatorname{Sym}(A, \sigma)=m^{2}+m(m+\varepsilon)=\frac{2 m(2 m+\varepsilon)}{2},
$$

where $m$ is the degree of $B$, and $\varepsilon= \pm 1$ the type of $\nu$. This proves that $\sigma$ also is of type $\varepsilon$.

By Lemma 1.6, the isomorphism class of $(A, \sigma)$ depends only on $(B, \tau), \beta$, and the type of $\sigma$; it does not depend on the particular choices of $u$ and $\nu$.

Example 1.12. If $(B, \tau)$ is hyperbolic (e.g., if $K=F \times F$ ), then $B$ contains an idempotent $e$ satisfying $\tau(e)=1-e$; the same equalities also hold in $A$, so $(A, \sigma)$ is hyperbolic [BST, 2.1].

Example 1.13. Assume that $(B, \tau)$ has a descent; that is, $B=B_{0} \otimes_{F} K$ and $\tau=\tau_{0} \otimes^{-}$for some central simple algebra $B_{0}$ over $F$ with involution $\tau_{0}$ of the first kind. (For example, this holds if $B$ is split.) Pick $\lambda \in F^{\times}$such that $\beta$ is the Brauer class of $B_{0} \otimes(K, \lambda)$. The algebra with involution $(B, \tau)$ has, up to isomorphism, exactly two extensions with Brauer class $\beta$ : one of orthogonal type and one of symplectic type. They both decompose as

$$
(A, \sigma)=\left(B_{0}, \tau_{0}\right) \otimes((K, \lambda), \gamma)
$$

where either $\gamma$ is the only orthogonal involution on $(K, \lambda)$ that acts as ${ }^{-}$on $K$, and $\sigma$ is of the same type as $\tau_{0}$, or $\gamma$ is the canonical involution on $(K, \lambda)$, and $\sigma$ and $\tau_{0}$ are of opposite type. This follows directly from Example 1.3 and Proposition 1.9.

The isomorphism class of the centralizer $B$ of $K$ in $A$ depends only on the isomorphism classes of $K$ and $A$ (and not on the embedding of $K$ in $A$ ) by SkolemNoether. On the other hand, using Example 1.13 , it is easy to construct an $(A, \sigma)$ even over a field of cohomological dimension 2-that contains two copies of $\left(K,{ }^{-}\right)$ such that the centralizers are not isomorphic as algebras with involution.

Remark 1.15. We now sketch the relationship between $(B, \tau)$ and $(A, \sigma)$ from the perspective of algebraic groups. Write $G$ for the group $\mathrm{SO}(A, \sigma)$ if $\sigma$ is orthogonal, resp. $\operatorname{Sp}(A, \sigma)$ if $\sigma$ is symplectic, and suppose that $K=F[\delta]$ is a field. Over a separable closure of $F$, the eigenspaces $V, W$ of $\delta$ are parallel totally isotropic subspaces. The stabilizers $P, Q$ of $V, W$ in $G$ are maximal parabolic subgroups defined over $K$. Their intersection consists of the elements of $G$ that stabilize both $V$ and $W$, i.e., that commute with $\delta$. It follows that the intersection $L:=P \cap Q$ is an $F$-defined subgroup of $G$. Its $F$-points are

$$
L(F)=\left\{b \in B \mid \tau(b) b=1_{B} \text { and } N_{K / F}\left(\operatorname{Nrd}_{B}(b)\right)=1_{F}\right\} .
$$

The group $L$ is reductive with center the norm 1 elements of $K^{\times}$and derived subgroup $\mathrm{SU}(B, \tau)$. Obviously, $L$ is a Levi subgroup of $P$ and $Q$; hence $P$ and $Q$ are opposite parabolics. Moreover, the nontrivial $F$-automorphism of $K$ interchanges the eigenspaces of $\delta$, hence also $P$ and $Q$.

In this section, we have described how to construct $(A, \sigma)$ from $(B, \tau)$; finding $(B, \tau)$ in $(A, \sigma)$ is a triviality given $K$. The second, easier direction is also standard from the viewpoint of algebraic groups: If we assume that $\sigma$ is hyperbolic over a quadratic extension $K / F$, then [PR, p. 383, Lemma $6.17^{\prime}$ ] gives the existence of opposite parabolic subgroups $P$ and $Q$ over $K$ like those in the previous paragraph. (But note that this result requires $P$ and $Q$ to be conjugate, so it does not apply in case $\sigma$ is orthogonal and $\operatorname{deg} A \equiv 2 \bmod 4$.) 


\section{ORthogonal EXTENSIONS OF $(B, \tau)$ AND THEIR INVARIANTS}

From now on, we consider algebras with orthogonal involution $(A, \sigma)$ that are quadratic extensions of some unitary $(B, \tau)$. Suppose further that $B$ has even degree, so that the degree of $A$ is divisible by 4 .

2.1. We claim that the discriminant of $\sigma$ is trivial and the class of the discriminant algebra of $(B, \tau)$ and the Clifford invariant of $(A, \sigma)$ agree in $H^{2}\left(F, \mu_{2}\right) /[A]$. It suffices to check these claims when $A$ is split, as can be seen by extending scalars to the function field of the Severi-Brauer variety of $A$. In that case, $(A, \sigma)$ is given by (1.14) where $B_{0}$ is split, and $\tau_{0}$ and $\gamma$ are orthogonal. The discriminant of $\sigma$ is obviously trivial. Further, the even Clifford algebra of $(A, \sigma)$ is $\left(K, \operatorname{disc} \tau_{0}\right)$ by $[\mathrm{L}$, V.3.15, V.3.16] or [KMRT, p. 147], and the discriminant algebra of $(B, \tau)$ is also Brauer-equivalent to $\left(K, \operatorname{disc} \tau_{0}\right)$ by [KMRT, 10.33].

Hence, $(A, \sigma)$ has trivial discriminant and Clifford invariant if and only if the discriminant algebra of $(B, \tau)$ is split or Brauer-equivalent to $A$. We have proved:

Lemma 2.2. For any algebra $(B, \tau)$ with unitary $K / F$ involution, of even degree and exponent at most 2 , the unique orthogonal quadratic extension of $(B, \tau)$ Brauerequivalent to the discriminant algebra $\mathcal{D}(B, \tau)$ has trivial discriminant and Clifford invariant.

The orthogonal quadratic extensions given by Lemma 2.2 can have index 1, 2, or 4 , and all three are possible. Indeed, the discriminant algebra $\mathcal{D}(B, \tau)$ has index dividing 4 [KMRT, 10.30]. Examples where $B$ has exponent 2 (as in Proposition 1.9) and $\mathcal{D}(B, \tau)$ has index 4 can be found in [KMRT, p. 145, Exercise 13] or can easily be constructed from Example1.13, even over some fields of cohomological dimension $\leq 2$.

Example 2.3. Suppose that $B$ is split and has even degree $n$, and the discriminant algebra $\mathcal{D}(B, \tau)$ is also split. Then $(B, \tau)$ descends as in Example 1.13, with $\tau_{0}$ adjoint to a quadratic form $\psi_{0} \cong\left\langle\alpha_{1}, \alpha_{2}, \ldots, \alpha_{n}\right\rangle$. As the discriminant algebra is split, the discriminant of $\psi_{0}$ is a norm from $K$. So the form $\psi:=\left\langle\left(\operatorname{disc} \psi_{0}\right) \alpha_{1}, \alpha_{2}, \ldots, \alpha_{n}\right\rangle$ is also a descent of $\tau$, i.e., $\operatorname{ad}_{\psi} \otimes^{-}$is an involution on $M_{n}(F) \otimes K$ isomorphic to $\tau$. Thus, the orthogonal quadratic extensions of $(B, \tau)$ can be decomposed as

$$
(A, \sigma)=\operatorname{Ad}_{\psi} \otimes(Q, \gamma)
$$

where $\psi$ has trivial discriminant, $Q$ is a quaternion algebra split by $K$, and $\gamma$ is the unique orthogonal involution on $Q$ whose restriction to $K$ is ${ }^{-}$.

By 2.1. those $(A, \sigma)$ have trivial discriminant and Clifford invariant. As the algebra has index 2, there is an Arason $e_{3}$-invariant defined for it via the method of $[\mathrm{Be}]$ or either of the methods in $\mathrm{GaO}$. We find

$$
e_{3}(A, \sigma)=[K] \cdot e_{2}(\psi) \quad \in H^{3}(F, \mathbb{Z} / 2 \mathbb{Z}) /\langle[Q]\rangle,
$$

for $\psi$ as in (2.4) and where $[K]$ denotes the class of $K$ in $H^{1}\left(F, \boldsymbol{\mu}_{2}\right)$ and $e_{2}(\psi)$ is the Clifford invariant of $\psi$. To prove this formula, one needs only check it when $Q$ is split. In that case, $\operatorname{ad}_{\psi} \otimes \gamma$ is adjoint to the quadratic form $\psi$ tensored with the norm form of the quadratic extension $K / F$, and the formula is clear. 


\section{Generalization of Pfister's theorem}

From any central simple algebra $(B, \tau)$ of even degree with unitary involution, Lemma 2.2 produces a central simple algebra of degree $2 n$ with trivial discriminant and Clifford invariant. We assert that for $n=6$, this construction produces all such algebras of degree 12 .

Theorem 3.1. Let $(A, \sigma)$ be a central simple algebra with orthogonal involution, where $A$ has degree 12. If $\sigma$ has trivial discriminant and Clifford invariant, then $(A, \sigma)$ is a quadratic extension of some central simple algebra $(B, \tau)$ of degree 6 and exponent at most 2 with unitary involution and such that the discriminant algebra $\mathcal{D}(B, \tau)$ is Brauer-equivalent to $A$.

It is an obvious corollary of the theorem that $(A, \sigma)$ is split and hyperbolic over an extension of degree dividing 4. Roughly speaking, this says that the torsion index of the half-spin group in dimension 12 is 4, a result of Totaro [To, 5.1].

Proof of Theorem 3.1. If $F$ is finite, then $A$ is split and $\sigma$ is hyperbolic, and we are done by Example 1.4. So assume that $F$ is infinite.

We first prove that $(A, \sigma)$ is a quadratic extension of some $(B, \tau)$ of degree 6 and exponent at most 2 , with no condition on the discriminant algebra. By [1.5, it suffices to prove that $\sigma$ is hyperbolic over $F$ or a quadratic extension of $F$. Indeed, if $A$ is split, then $\sigma$ is adjoint to a 12-dimensional quadratic form $q$ in $I^{3}$, which cannot have odd Witt index by Pfister's theorem for 10-dimensional quadratic forms in $I^{3}$ and the Arason-Pfister Hauptsatz.

As $(A, \sigma)$ has trivial discriminant and Clifford invariant, one of the half-spin representations $V$ of $\operatorname{Spin}(A, \sigma)$ is defined over $F$. Over an algebraic closure $F_{\text {alg }}$, the group $\operatorname{Spin}(A, \sigma)$ has an open orbit $\mathcal{O}$ in $\mathbb{P}(V)\left(F_{\text {alg }}\right)$; see $\mathbb{I}$, p. 1012] or [GaC]. But $F$ is infinite, so there is some $v \in V$ over $F$ such that $[v]$ belongs to $\mathcal{O}$.

We consider the image in $\operatorname{SO}(A, \sigma)$ of the stabilizer of $[v]$ in $\operatorname{Spin}(A, \sigma)$. Its identity component $H$ is isomorphic over $F_{\text {alg }}$ to $\mathrm{SL}_{6}$ by [I]. Indeed, over $F_{\text {alg, we }}$ may identify $\mathrm{SO}(A, \sigma)$ with the special orthogonal group $\mathrm{SO}_{12}$ of the symmetric bilinear form

$$
(x, y) \mapsto x^{t}\left(\begin{array}{cc}
0 & 1_{6} \\
1_{6} & 0
\end{array}\right) y,
$$

where $1_{6}$ denotes the 6 -by- 6 identity matrix. As $[v]$ belongs to the orbit $\mathcal{O}$, up to conjugacy $H$ is the copy of $\mathrm{SL}_{6}$ in $\mathrm{SO}_{12}$ given by the inclusion

$$
a \mapsto\left(\begin{array}{cc}
a & 0 \\
0 & a^{-t}
\end{array}\right) \text {. }
$$

From this, we see that the natural 12-dimensional representation of $\mathrm{SO}_{12}$ decomposes as a direct sum of inequivalent 6-dimensional representations of $\mathrm{SL}_{6}$. The centralizer $C$ of $H$ in $\mathrm{SO}(A, \sigma)$ is a rank 1 torus; it consists over $F_{\text {alg }}$ of the matrices

$$
\left(\begin{array}{cc}
\alpha 1_{6} & 0 \\
0 & \alpha^{-1} 1_{6}
\end{array}\right) \text { for } \alpha \in F_{\text {alg }}^{\times} .
$$

So there is a field $K / F$ such that $[K: F]=1$ or 2 that splits $C$. The centralizer of $C(F)$ in $A \otimes K$ is (as can be seen over $F_{\text {alg }}$ ) a product of central simple algebras $B_{+} \times B_{-}$, where $B_{+}$and $B_{-}$have degree 6 and $\sigma$ interchanges the two factors. Put $\delta:=(1,-1)$ in $B_{+} \times B_{-}$. Obviously $\delta^{2}=1$ and $\sigma(\delta)=-\delta$, because these equations hold over $F_{\text {alg. It follows that }}(A, \sigma)$ is hyperbolic over $K$.

We have proved that $(A, \sigma)$ is a quadratic extension of some $(B, \tau)$ with unitary $K / F$ involution. Moreover, by 2.1 the discriminant algebra $\mathcal{D}(B, \tau)$ has Brauer 
class $[A]$ or 0 . Hence we are done except if $\mathcal{D}(B, \tau)$ is split and $A$ is nonsplit. If so, the algebra $B$ is split by KMRT, 10.30], and $(A, \sigma)$ is of the special form described in Example 2.3. We view $Q$ as generated by pure quaternions $\delta$ and $j$ that anti-commute and satisfy $\delta^{2}=d$ and $j^{2}=\lambda$ for some $\lambda \in F^{\times}$with $Q \cong(K, \lambda)$. Let $\psi=\left\langle\alpha_{1}, \alpha_{2}, \ldots, \alpha_{6}\right\rangle$ be the quadratic form of discriminant 1 as in equation (2.4). It naturally gives a $\gamma$-hermitian form on $Q$. Scaling the first basis vector (of length $\alpha_{1}$ ) by $j$ gives an isomorphic $\gamma$-hermitian form and changes the $\alpha_{1}$ in the diagonalization to $\gamma(j) \alpha_{1} j=j^{2} \alpha_{1}=\lambda \alpha_{1}$; this form is obtained from the quadratic form $\psi^{\prime}=\left\langle\lambda \alpha_{1}, \alpha_{2}, \ldots, \alpha_{6}\right\rangle$. That is,

$$
(A, \sigma) \cong \operatorname{Ad}_{\psi} \otimes(Q, \gamma) \cong \operatorname{Ad}_{\psi^{\prime}} \otimes(Q, \gamma)
$$

So $(A, \sigma)$ can also be constructed from $\left(B, \tau^{\prime}\right)=\operatorname{Ad}_{\psi^{\prime}} \otimes\left(K,{ }^{-}\right)$, as in Example 1.13. for which the discriminant algebra is Brauer-equivalent to $\left(K, \operatorname{disc} \psi^{\prime}\right)=(K, \lambda)=$ $Q$.

Corollary 3.2 (Pfister's Theorem). If $q$ is a 12-dimensional form with trivial discriminant and Clifford invariant, then $q$ is isomorphic to $\phi \otimes \psi$ for some 1-Pfister form $\phi$ and 6-dimensional quadratic form $\psi$ with trivial discriminant.

Proof. By Theorem 3.1, $\left(M_{12}(F), \operatorname{ad}_{q}\right)$ is a quadratic extension of $\left(M_{6}(K), \tau\right)$ for some quadratic étale $F$-algebra $K$ and unitary involution $\tau$ with split discriminant algebra. By Example 2.3 and specifically (2.4), $q$ is similar to the tensor product of the norm form of $K / F$ (call it $\phi$ ) with a 6-dimensional quadratic form $\psi$ of discriminant 1 . Replacing $\psi$ with a multiple does not change the discriminant, and it completes the proof.

Remark 3.3. Theorem 3.1 says something about groups $G$ of type $E_{7}$ whose Tits index (as defined in Ti66]) is

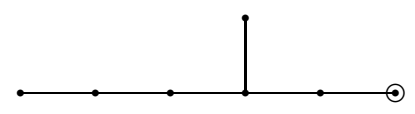

or has more vertices circled. For such a $G$, the obvious subdiagram of type $D_{6}$ corresponds to a semisimple subgroup $H$ of type $D_{6}$, and the isogeny class of $H$ determines $G$ by Tits's Witt-type theorem [Ti66, Remark 2.7.2(d)]. It follows from Ti71, §5] that $H$ is isogenous to $\operatorname{SO}(A, \sigma)$, where $(A, \sigma)$ is as in Theorem 3.1.

We remark that such groups $G$ are the only remaining open case of the KneserTits Problem over fields of cohomological dimension $\leq 2$; see [Gil, 8.6].

\section{ACKNOWLEDGMENTS}

The first author's research was partially supported by National Science Foundation grant DMS-0654502. Both authors would like to thank the Institut des Hautes Études Scientifiques for a pleasant working environment while some of the research for this paper was performed.

\section{REFERENCES}

[A] A.A. Albert, Structure of algebras, AMS Coll. Pub., vol. 24, AMS, Providence, RI, 1961, revised printing. MR0123587 (23:A912)

[BP] E. Bayer-Fluckiger and R. Parimala, Galois cohomology of the classical groups over fields of cohomological dimension $\leq 2$, Invent. Math. 122 (1995), 195-229. MR1358975 (96i:11042) 
[BST] E. Bayer-Fluckiger, D.B. Shapiro, and J.-P. Tignol, Hyperbolic involutions, Math. Z. 214 (1993), no. 3, 461-476. MR.1245206 (94j:16060)

[Be] G. Berhuy, Cohomological invariants of quaternionic skew-Hermitian forms, Arch. Math. (Basel) 88 (2007), no. 5, 434-447. MR2316889 (2008d:12005)

[D] J. Dieudonné, Les extensions quadratiques des corps non commutatifs et leurs applications, Acta Math. 87 (1952), 175-242. MR0049873 (14:239e)

[ET] M.A. Elomary and J.-P. Tignol, Classification of quadratic forms over skew fields of characteristic 2, J. Algebra 240 (2001), 366-392. MR.1830558 (2002f:11039)

[GaC] S. Garibaldi, Cohomological invariants: Exceptional groups and spin groups, with an appendix by Detlev W. Hoffmann, Memoirs Amer. Math. Soc., to appear.

[GaO] - Orthogonal involutions on algebras of degree 16 and the Killing form of $E_{8}$, with an appendix by Kirill Zainoulline, preprint, 2008.

[Gi] P. Gille, Le problème de Kneser-Tits, Séminaire Bourbaki, 60ème année, no. 983, 20062007.

[I] J.-I. Igusa, A classification of spinors up to dimension twelve, Amer. J. Math. 92 (1970), 997-1028. MR0277558 (43:3291)

[KMRT] M.-A. Knus, A.S. Merkurjev, M. Rost, and J.-P. Tignol, The book of involutions, Colloquium Publications, vol. 44, Amer. Math. Soc., Providence, RI, 1998. MR 1632779 (2000a:16031)

[L] T.Y. Lam, Introduction to quadratic forms over fields, Graduate Studies in Mathematics, vol. 67, Amer. Math. Soc., Providence, RI, 2005. MR2104929 (2005h:11075)

[Pf] A. Pfister, Quadratische Formen in beliebigen Körpern, Invent. Math. 1 (1966), 116-132. MR.0200270 (34:169)

[PR] V.P. Platonov and A. Rapinchuk, Algebraic groups and number theory, Academic Press Inc., Boston, MA, 1994. MR1278263 (95b:11039)

[Q] A. Quéguiner-Mathieu, Invariants cohomologiques: des formes quadratiques aux algèbres à involution, Théorie des nombres (Besançon, 2002), Publ. Math. UFR Sci. Tech. Besançon, Univ. France-Comté, 2002. MR 1990438 (2004h:11033)

[QT] A. Quéguiner-Mathieu and J.-P. Tignol, Discriminant and Clifford algebras, Math. Zeit. 240 (2002), 345-384. MR.1900315 (2003e:16023)

[R] M. Rost, On 14-dimensional quadratic forms, their spinors, and the difference of two octonion algebras, preprint, March 1999.

[SK] M. Sato and T. Kimura, A classification of irreducible prehomogeneous vector spaces and their relative invariants, Nagoya Math. J. 65 (1977), 1-155. MR0430336 (55:3341)

[Ti66] J. Tits, Classification of algebraic semisimple groups, Algebraic Groups and Discontinuous Subgroups, Proc. Symp. Pure Math., vol. IX, Amer. Math. Soc., Providence, RI, 1966, pp. 32-62. MR0224710 (37:309)

[Ti71] L Représentations linéaires irréductibles d'un groupe réductif sur un corps quelconque, J. Reine Angew. Math. 247 (1971), 196-220. MR0277536 (43:3269)

[To] B. Totaro, The torsion index of $E_{8}$ and other groups, Duke Math. J. 129 (2005), 219248. MR2165542(2006f:57039a)

Department of Mathematics and Computer Science, Emory University, Atlanta, GEORGIA 30322

E-mail address: skip@member.ams.org

URL: http://www.mathcs.emory.edu/ ${ }^{\sim}$ skip/

Université Paris 13 (LAGA), CNRS (UMR 7539), Université Paris 12 (IUFM), 93430 Villetaneuse, France

E-mail address: queguin@math.univ-paris13.fr

$U R L:$ http://www-math.univ-paris13.fr/ queguin/ 